\title{
Push-Out Bond Strength and Surface Microhardness of Calcium Silicate-Based Biomaterials: An in vitro Study
}

\author{
Abdul Majeed Emad AlShwaimi \\ Division of Endodontics, Restorative Dental Sciences Department, College of Dentistry, University of Dammam, \\ Dammam, Saudi Arabia
}

\section{Significance of the Study}

In this study, the push-out bond strength and microhardness of 3 materials, ProRoot MTA, Biodentine, and BioAggregate, were compared. Biodentine and ProRoot MTA had higher bond strength and microhardness values than BioAggregate, and hence could be the materials of choice for root repair procedures and retrograde fillings.

\section{Keywords}

Calcium silicate-based materials · Mineral trioxide aggregate $\cdot$ Push-out bond strength · Root repair materials . Surface microhardness

\begin{abstract}
Objective: This was an in vitro evaluation of push-out bond strength and surface microhardness of calcium silicatebased biomaterials in coronal and apical root dentin. Materials and Methods: Ninety sections ( $2 \mathrm{~mm}$ thick) of coronal and apical root dentin were obtained from roots of 60 extracted teeth; the canals were enlarged to a standardized cavity diameter of $1.3 \mathrm{~mm}$. Sections were randomly divided into 6 groups ( $n=15$ per group), and cavities were filled with Biodentine $^{\mathrm{TM}}$, BioAggregate, or ProRoot mineral trioxide aggregate (MTA), according to the manufacturers' instructions. Push-out bond strength values were measured using a universal testing machine under a compressive load at a speed
\end{abstract}

\begin{tabular}{ll}
\hline KARGER & ( 2016 S. Karger AG, Basel \\
$\begin{array}{l}\text { E-Mail karger@karger.com } \\
\text { www.karger.com/mpp }\end{array}$ & $\begin{array}{l}\text { This is an Open Access article licensed under the terms of the } \\
\text { Creative Commons Attribution-NonCommercial } 3.0 \text { Un- } \\
\text { ported license (CC BY-NC) (www.karger.com/OA-license), } \\
\text { applicable to the online version of the article only. Distribu- } \\
\text { tion permitted for non-commercial purposes only. }\end{array}$
\end{tabular}

of $1 \mathrm{~mm} / \mathrm{min}$. Samples were analyzed under a light microscope to determine the nature of bond failure. Ten samples ( $2 \mathrm{~mm}$ thick) were prepared for all the materials, and Vickers microhardness was determined using a digital hardness tester. Data were analyzed using one-way analysis of variance and Tukey-Kramer multiple comparison tests at a significance level of $p<0.05$. Results: Biodentine (42.02; 39.35 MPa) and ProRoot MTA (21.86; 34.13 MPa) showed significantly higher bond strengths than BioAggregate $(6.63 ; 10.09$ $\mathrm{MPa}$ ) in coronal and apical root dentin, respectively ( $p<$ 0.05). Biodentine also differed significantly from ProRoot MTA in coronal dentin. Bond failure was predominantly adhesive in Biodentine and ProRoot MTA, while BioAggregate showed predominantly mixed failure. ProRoot MTA (158.52 $\mathrm{HV}$ ) showed significantly higher microhardness and BioAggregate (68.79 HV) showed the lowest hardness. Conclusion: Biodentine and ProRoot MTA showed higher bond strength and microhardness compared to BioAggregate.

(c) 2016 S. Karger AG, Basel

Assoc. Prof. Emad AlShwaimi, BDS, FRCD(C), DMSc

Division of Endodontics, Restorative Dental Sciences Department

College of Dentistry, University of Dammam

PO Box 1982, Dammam 31441 (Saudi Arabia)

E-Mail ealshwaimi@uod.edu.sa 


\section{Introduction}

Bacteria and their by-products are the main causes of periapical inflammation in endodontics [1]. The purpose of endodontic therapy is to clean and disinfect the root canal system with subsequent filling to provide an impervious apical seal [1]. In some cases, if conventional endodontic therapy fails and/or retreatment is not feasible, apicoectomy is performed and a root-end filling is placed to seal the apex [2]. On the other hand, the quality of coronal restoration has been reported to be more important than the quality of obturation itself in maintaining a healthy periapex [3]. Saunders and Saunders [4] reported that poor coronal sealing was a major cause of nonsurgical endodontic treatment failure. The placement of an intraorifice barrier immediately following root filling could reduce coronal leakage in root-canal-treated teeth [5].

A number of different materials have been tested in order to determine their ability to provide a coronal and/ or apical seal. Mineral trioxide aggregate (MTA), a calcium silicate-based cement, has rapidly gained popularity due to its ability to resist leakage and its superior marginal adaptation to the dentinal walls [1]. It is composed of tricalcium silicate, dicalcium silicate, bismuth oxide, tricalcium aluminate, and gypsum [6]. It has been used in numerous clinical situations such as direct pulp capping, repair of root perforations, apexogenesis, apexification, and as a root-end filling material [7]. In addition, it is used as a coronal barrier following regenerative endodontic treatment [8]. However, MTA has some disadvantages such as a long setting time, poor handling, and a high price [9].

Recently, 2 new calcium silicate-based cements were introduced into the market $[6,10]$. The first was Biodentine $^{\mathrm{TM}}$ (Septodont, Saint Maur des Fossés, France), which is composed mainly of purified tricalcium silicate powder, small amounts of dicalcium silicate, calcium carbonate, and an opaque calcium chloride liquid [10]. The second, BioAggregate (Innovative Bioceramix, Vancouver, BC, Canada), is also composed of tricalcium silicate, dicalcium silicate calcium hydroxide, and hydroxyapatite [6]. Both materials are recommended for direct pulp capping, repair of perforations, apexogenesis, apexification, and as root-end fillings.

Ideal cement for use in any of the above situations is expected to have adequate physicochemical properties and withstand the dislodging forces that occur as a result of tooth function or operative procedures [11]. Surface microhardness has been used as an indicator of the overall strength of a material and its performance under mas- ticatory stresses [12]. For hydraulic cements such as MTA, surface microhardness depicts the degree of hydration that occurred during the setting process and the influence of the setting environment on the overall strength of the material [13]. However, push-out bond strength testing is a practical and reliable method to evaluate the adaptation of a material to its surrounding root dentin [14]. A few studies $[6,15,16]$ have evaluated the push-out bond strengths of MTA, Biodentine, and BioAggregate, but no report has compared all 3 of these materials. Saghiri et al. [6] reported significantly higher push-out bond strength values for nano-white MTA compared to white MTA and BioAggregate, while other studies reported higher bond strength values for Biodentine compared to conventional root perforation repair materials $[15,16]$. Camilleri et al. [17] reported that small differences in the composition of new generation tricalcium silicate-based material such as BioAggregate modify the setting kinetics and thus the end product of hydration.

Cement materials come in to contact with the coronal and apical root dentin when they are used as intraorifice barriers or root-end fillings, respectively. Therefore, the aim of this study was to evaluate the push-out bond strength and surface microhardness of Biodentine, BioAggregate, and ProRoot MTA in both coronal and apical root dentin.

\section{Materials and Methods}

The study was approved by the Institutional Review Board, Deanship of Scientific Research of the University of Dammam, and it was conducted in full accordance with the World Medical Association Declaration of Helsinki. Eighty single-rooted human teeth were collected from the Oral Surgery Department of the College of Dentistry. Teeth were extracted for periodontal or orthodontic reasons not related to this study. Teeth were cleaned of remaining tissue and calculus, and stored in 1\% thymol solution. Teeth with caries, curved roots, open apices, cracks, or previous root canal treatments were excluded. The crowns of all teeth were sectioned close to the cementum-enamel junction using a diamond bur. Teeth were embedded in epoxy resin, and 3-mm sections were removed from the coronal and apical root areas. The remaining roots of the teeth were cut horizontally (transversely) using a water-cooled low-speed diamond saw (Isomet; Buehler, Lake Bluff, NY, USA) to obtain 90 dentin sections of $2 \mathrm{~mm}$ in thickness from the coronal and apical thirds of each root. Both coronal and apical root dentin sections were kept in separate containers. The canals of all dentin sections were enlarged to a standardized cavity size of $1.3 \mathrm{~mm}$ in diameter using a round diamond bur and No. 5 Gates-Glidden drills. Each diamond bur and GatesGlidden drill was replaced after every 5 preparations. All sections were immersed in 17\% EDTA solution for $3 \mathrm{~min}$ to remove the smear layer, followed by immersion in $1.5 \%$ sodium hypochlorite 
Fig. 1. Photograph showing the placement of custom-made jig with specimen aligned to the stainless steel plunger before pushout bond test (a) and fractured tested material being pushed out of the test cavity (b).
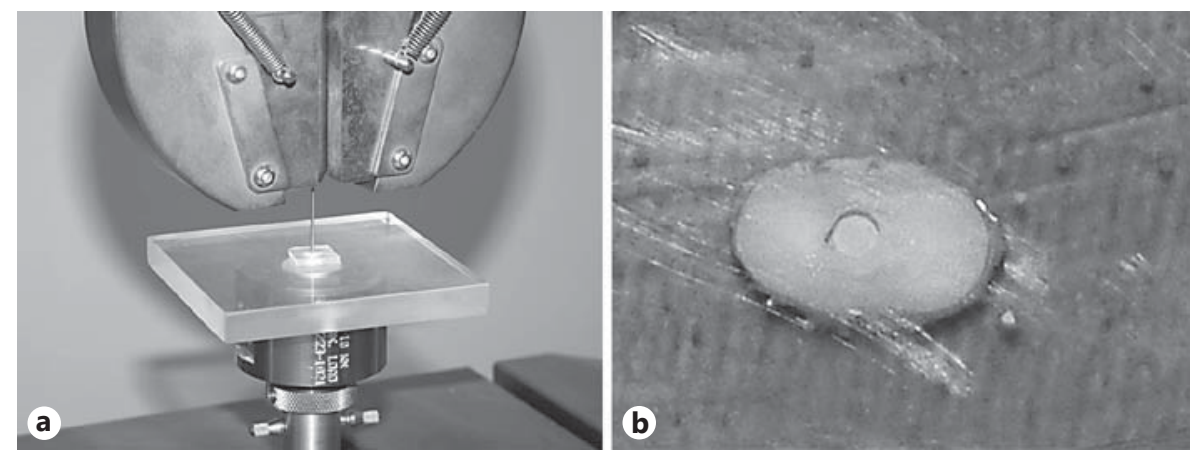

solution for $3 \mathrm{~min}$. Sections were rinsed thoroughly with distilled water and dried using gauze pieces. Both coronal and apical root dentin sections were randomly divided into 6 groups $(n=15$ per group) and the cavities were filled as follows.

\section{Coronal Root Dentin}

Group 1: Biodentine (Septodont, B06955). Biodentine was mixed according to the manufacturer's instructions. First, the powder capsule was opened and placed in a container, and then the entire volume of liquid from the single dose container was poured into the powder. The powder capsule was closed and mixed for $30 \mathrm{~s}$ using an amalgamator. The mixed Biodentine material was collected using an amalgam carrier and placed into the cavities. Dentin sections were left in place for $10 \mathrm{~min}$ to allow for the initial setting of the material. After this, the specimens were wrapped in a gauze piece soaked in artificial saliva and stored in an incubator at $37^{\circ} \mathrm{C}$ for $72 \mathrm{~h}$.

Group 2: White ProRoot ${ }^{\circledR}$ MTA (Dentsply Tulsa Dental Specialties, Tulsa, OK, USA: 11004374E). ProRoot MTA was mixed according to the manufacturer's instructions. First, the powder was dispensed onto a mixing pad and then the entire volume of the liquid microdose ampule was poured onto the mixing pad next to the powder. The powder and liquid were mixed thoroughly for $1 \mathrm{~min}$ using the mixing stick provided until a thick creamy mixture was formed. The mixed ProRoot MTA material was collected using an amalgam carrier and placed into the cavities. Dentin sections were left in place for 5-10 min to allow for the initial setting of the material. After this, the specimens were wrapped in a gauze piece soaked in artificial saliva and stored in an incubator at $37^{\circ} \mathrm{C}$ for $72 \mathrm{~h}$.

Group 3: BioAggregate ${ }^{\circledR}$ (Innovative Bioceramix Inc., 1201BA). BioAggregate was mixed according to the manufacturer's instructions. First, the powder was dispensed into a mixing cup and then the entire volume of liquid from a vial of BioAggregate liquid was poured into the powder. The powder and liquid were mixed thoroughly for 2 min using the spatula provided until a thick paste-like mixture was formed. The mixed BioAggregate material was collected using an amalgam carrier and placed into the cavities. Dentin sections were left in place for 5-10 min to allow for the initial setting of the material. After this, the specimens were wrapped in a gauze piece soaked in artificial saliva and stored in an incubator at $37^{\circ} \mathrm{C}$ for $72 \mathrm{~h}$.

\section{Apical Root Dentin}

Group 4: Biodentine. The procedure for material application and sample preparation was the same as that described above for group 1 .

Bond Strength of Root Repair Materials
Group 5: ProRoot MTA. The procedure for material application and sample preparation was the same as that described above for group 2.

Group 6: BioAggregate. The procedure for material application and sample preparation was the same as that described above for group 3 .

\section{Push-Out Bond Strength Evaluation}

Push-out bond strength was evaluated for all samples using a Zwick/Roell Z050 universal testing machine (Zwick GmbH \& Co., Ulm, Germany). Samples were placed on a custom-made slab with a hole in the center. The hole was aligned with the center of the test specimen. This allowed the stainless steel needle of the testing machine to pass through freely once the bond between the test material and the root dentin wall was broken. A stainless steel needle of $1 \mathrm{~mm}$ in diameter was used to apply a compressive load with downward pressure on the surface of the tested material at a speed of $1 \mathrm{~mm} / \mathrm{min}$ [6]. The needle was placed in the center of the test material so that it had $0.2 \mathrm{~mm}$ of clearance from the dentin wall (Fig. 1). The maximum load at which the specimen was dislodged was recorded in Newtons $(\mathrm{N})$. The bond strength was calculated in MPa using the following formula:

$$
\text { bond strength }(\mathrm{MPa})=\frac{\text { debonding force }(\mathrm{N})}{\text { surface area }\left(\mathrm{mm}^{2}\right)} \text {. }
$$

\section{Surface Microhardness}

Ten specimens of each material were prepared using a $5-\mathrm{mm}$ wide and 2-mm high mold, according to the manufacturers' instructions. Following $72 \mathrm{~h}$ of storage in $100 \%$ relative humidity at $37^{\circ} \mathrm{C}$, the outer surfaces of all specimens were polished with watercooled carbide paper of up to 1,200 grit fineness (3M, St. Paul, MN, USA) using a universal polisher (Metaserv, Betchworth, Surrey, UK). Surface microhardness was measured using an automatic Vickers microhardness tester (FM-ARS 9000; Future-Tech Corp, Kawasaki, Japan). Five indents were made on the surface of each sample with a 25-g load applied for 5 s [18]. Values were recorded as Vickers hardness (HV) in an MS Excel spreadsheet.

\section{Data Analysis}

Push-out bond strength was the main outcome tested in this study. NCSS 2007 software (NCSS LLC, Kaysville, UT, USA) was used for the statistical analysis of both push-out bond strength and surface microhardness. One-way analysis of variance followed by Tukey-Kramer multiple comparison tests were used at a signifi- 
Table 1. Mean push-out bond strength values with standard deviations and nature of bond failure for all groups

\begin{tabular}{|c|c|c|c|c|c|c|}
\hline \multirow[t]{2}{*}{ No. } & \multirow[t]{2}{*}{ Groups } & \multicolumn{2}{|c|}{ Mean bond strength values, $\mathrm{MPa}$} & \multicolumn{3}{|c|}{ Nature of bond failure, $\%$} \\
\hline & & coronal dentin & apical dentin & adhesive & cohesive & mixed \\
\hline 1 & Biodentine & $42.02 \pm 14.48^{\mathrm{A}, \mathrm{a}}$ & $39.35 \pm 16.22^{\mathrm{A}, \mathrm{a}}$ & 70 & - & 30 \\
\hline 2 & ProRoot MTA & $21.86 \pm 10.10^{\mathrm{A}, \mathrm{b}}$ & $34.13 \pm 7.97^{\mathrm{A}, \mathrm{a}}$ & 85 & - & 15 \\
\hline 3 & BioAggregate & $6.63 \pm 2.52^{\mathrm{A}, \mathrm{c}}$ & $10.09 \pm 4.22^{\mathrm{A}, \mathrm{b}}$ & - & 20 & 80 \\
\hline
\end{tabular}

Different superscript uppercase letters (row) or lowercase letters (column) represent significant differences $(p<0.01)$.

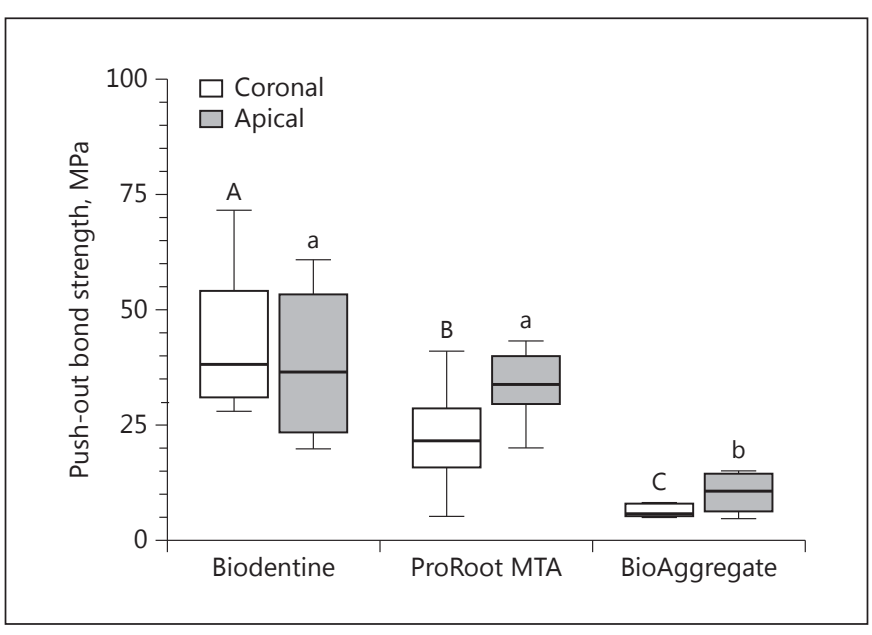

Fig. 2. Box-and-whisker plot showing median push-out bond strength values $(\mathrm{MPa})$ and variations for all groups in coronal and apical dentin. Different uppercase letters (coronal dentin) and lowercase letters (apical dentin) are significant differences amongst different materials $(p<0.05)$.

cance level of $p<0.05$. A Wilcoxon rank-sum test was used to compare the bond strength values between coronal and apical root sections within each group $(p<0.05)$.

\section{Results}

\section{Push-Out Bond Strength}

The mean bond strength values for all groups are given in Table 1. The box-and-whisker plots for bond strength values in coronal and apical dentin are shown in Figure 2.

Coronal Root Dentin. The Biodentine (42.02 MPa) group showed a significantly higher push-out bond strength than the ProRoot MTA (21.86 MPa) and BioAg- gregate $(6.63 \mathrm{MPa})$ groups in coronal dentin specimens $(p<0.05)$. BioAggregate produced the lowest bond strength and differed significantly from ProRoot MTA $(p<0.05$; Table 1, Fig. 2).

Apical Root Dentin. Push-out bond strength values for Biodentine (39.35 MPa) and ProRoot MTA (34.13 MPa) were significantly higher when compared to the BioAggregate $(10.09 \mathrm{MPa})$ group $(p<0.05)$. No statistically significant differences were found between ProRoot MTA and Biodentine in apical dentin ( $p>0.05$; Table 1, Fig. 2). Biodentine showed similar bond strength in coronal and apical root dentin while ProRoot MTA and BioAggregate showed slightly higher bond strength in apical root dentin than coronal dentin. However, the differences were not statistically significant for all 3 material groups $(p>$ 0.05; Table 1).

Nature of Bond Failure. The majority of samples in the Biodentine (70\%) and ProRoot MTA (85\%) groups showed adhesive-type bond failure, while the BioAggregate group exhibited $80 \%$ mixed (adhesive and cohesive) failure (Table 1).

\section{Surface Microhardness}

The mean surface microhardness values for all groups were as follows: ProRoot MTA $=158.52 \pm 18.43 \mathrm{HV}$, Biodentine $=115.87 \pm 7.43 \mathrm{HV}$, and BioAggregate $=68.79 \pm$ 13.34 HV. The differences were statistically significant among the groups $(p<0.05)$. Figure 3 shows a box-andwhisker plot for surface microhardness of all tested materials.

Surface microhardness for the ProRoot MTA (158.52 $\mathrm{HV})$ group was significantly higher compared to the Biodentine (115.87 HV) and BioAggregate (68.79 HV) groups $(p<0.05)$. The BioAggregate group showed the lowest hardness values and differed significantly from the Biodentine group $(p<0.05)$. 


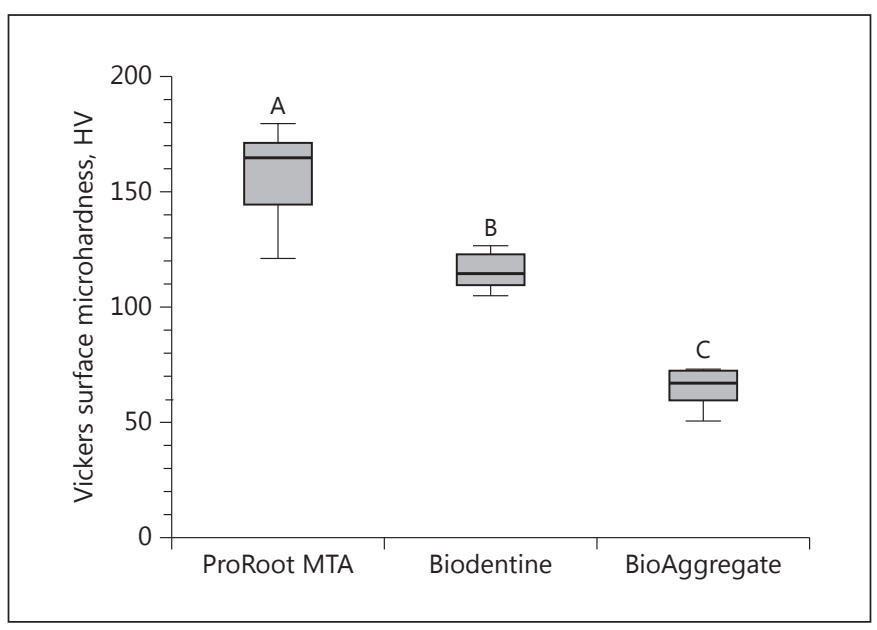

Fig. 3. Box-and-whisker plot showing surface microhardness for all groups. Different uppercase letters represent significant differences $(p<0.05)$.

\section{Discussion}

In this study, the bond strength values of Biodentine and ProRoot MTA in both coronal and apical dentin were higher than those of BioAggregate material; however, ProRoot MTA and BioAggregate had slightly lower values in coronal dentin than in apical dentin, but these differences were not statistically significant. Biodentine also had significantly higher bond strength than ProRoot MTA in coronal dentin. These findings are similar to those of previous studies [16, 19, 20]. Guneser et al. [16] demonstrated that Biodentine showed significantly higher bond strength than MTA, and exposure to various endodontic irrigants did not affect this finding negatively. Alsubait et al. [19] reported that BioAggregate showed significantly lower bond strength compared to ProRoot MTA and Biodentine. Recently, Dawood et al. [20] reported that the push-out bond strength values of Biodentine were higher than those of ProRoot MTA. In a previous study, Saghiri et al. [6] also reported significantly higher push-out bond strength values for nano-MTA and MTA as compared to BioAggregate cement.

The differences in the bond strengths of these materials could be due to their composition as previously reported [21, 22]. Being active biomaterials, calcium silicate-based cements produce calcium phosphate and apatite-like precipitates at the cement-dentin interface and within the dentinal tubules. This results in the formation of tag-like structures and an interfacial hybrid layer that is responsible for chemical and mechanical bonding [10].
The biomineralization ability of calcium silicate-based cements is directly proportional to the amount of $\mathrm{Ca}^{2+}$ released by them and the presence of phosphate in the tissue fluids [21]. The higher bond strength values of Biodentine observed in the present study could be attributed to its higher content of calcium-releasing products triggering the formation of tag-like structures at the cementdentin interface, resulting in increased resistance to dislodgement forces when compared to ProRoot MTA and BioAggregate $[22,23]$. On the other hand, BioAggregate did not contain aluminum compounds, but had other additives such as calcium phosphate and silicon dioxide. BioAggregate has been reported to react more slowly compared to other tricalcium silicate-based materials and did not show formation of calcium hydroxide and leaching of calcium ions in aged material [17]. BioAggregate also has a higher leaching rate of calcium ions that peaks after 1 day compared to MTA which shows low levels of calcium ions during the early stages of hydration and continues to increase as the material ages. The early higher leaching rate of BioAggregate could result in higher porosity of the set material and weak physical properties [17]. Equally important, it has been reported that BioAggregate has low compressive strength and surface microhardness compared to other root-end filling materials such as Biodentine and IRM $[17,24]$. Camilleri et al. [17] suggested the addition of a water-soluble polymer to improve the physical properties of BioAggregate.

In the current study, bond failures were predominantly adhesive in nature for Biodentine and ProRoot MTA, and they were predominantly mixed adhesive and cohesive in nature for BioAggregate. Adhesive bond failures in the Biodentine group were slightly less common when compared to ProRoot MTA. These findings are partly in agreement with previous studies that reported adhesive failures for ProRoot MTA and cohesive failures for Biodentine $[6,16,20]$. The predominantly mixed failures observed for BioAggregate could have been due to weak cement or incomplete setting resulting in poor physical properties. Differences in the particle size of the materials tested affect their penetration into the dentinal tubules and could be related to the differences in bond strength and the nature of bond failure recorded [6]. Biodentine is a manufactured material with a small particle size that promotes better penetration and micromechanical adhesion to the dentin compared to naturally derived ProRoot MTA [10, 22].

Although the sample preparation provided a canal space perfectly perpendicular to the applied load and prevented any frictional resistance [25] that could influence 
the results, differences were observed in the bond strengths of 2 tested materials between coronal and apical dentin. In addition to the differences in the composition of tested materials, this could be related to the variations in the dentin structure from the coronal to the apical area [26] because the adhesiveness of a material is directly dependent upon its interaction with the dentin surface [27]. However, previous studies on bond strength evaluation have not taken this into consideration. For example, as a rule, previous studies have utilized mid-root sections of dentin for evaluating push-out bond strength of root-end filling materials $[6,16]$. Therefore, the resulting bond strengths may have been under- or overestimated due to differences in the structure of the test substrate [28].

In the present study, surface microhardness of ProRoot MTA was significantly higher compared to Biodentine and BioAggregate biomaterials. The BioAggregate group showed the lowest hardness values and differed significantly from other groups. The findings are in agreement with the results of the previous studies $[29,30]$. Bolhari et al. [29] reported significantly higher hardness values for ProRoot MTA compared to BioAggregate and calcium-enriched material. The low surface microhardness of BioAggregate could be related to the increased initial leaching of calcium ions resulting in high porosity of the set material and weak physical properties [17]. In another study, Caronna et al. [30] reported significantly higher surface microhardness for ProRoot MTA when compared to EndoSequence Root Repair Material and Biodentine.

\section{Conclusion}

Biodentine and ProRoot MTA exhibited comparable bond strength values in apical root dentin with a similar nature of bond failure. However, a significantly higher displacement force was required to break the bond in coronal dentin in the Biodentine group. The surface microhardness of ProRoot MTA was significantly higher when compared to Biodentine. On the other hand, BioAggregate showed the lowest bond strength values as well as the lowest surface microhardness as compared to the other 2 materials.

\section{References}

1 Yavari H, Samiei M, Eskandarinezhad M, et al: An in vitro comparison of coronal microleakage of three orifice barriers filling materials. Iran Endod J 2012;7:156-160.

2 Post LK, Lima FG, Xavier CB, et al: Sealing ability of MTA and amalgam in different root-end preparations and resection bevel angles: an in vitro evaluation using marginal dye leakage. Braz Dent J 2010;21:416-419.

3 Ray HA, Trope M: Periapical status of endodontically treated teeth in relation to the technical quality of the root filling and the coronal restoration. Int Endod J 1995;28: 12-18.

4 Saunders WP, Saunders EM: Coronal leakage as a cause of failure in root-canal therapy: a review. Endod Dent Traumatol 1994;10:105108.

5 Roghanizad N, Jones JJ: Evaluation of coronal microleakage after endodontic treatment. J Endod 1996;22:471-473.

6 Saghiri MA, Garcia-Godoy F, Gutmann JL, et al: Push-out bond strength of a nano-modified mineral trioxide aggregate. Dent Traumatol 2013;29:323-327.

7 Noetzel J, Ozer K, Reisshauer BH, et al: Tissue responses to an experimental calcium phosphate cement and mineral trioxide aggregate as materials for furcation perforation repair: a histological study in dogs. Clin Oral Investig 2006; 10:77-83.
8 Dawood AE, Parashos P, Wong RH, et al: Calcium silicate-based cements: composition, properties, and clinical applications. J Investig Clin Dent 2015, DOI: 10.1111/jicd. 12195.

9 Ber BS, Hatton JF, Stewart GP: Chemical modification of ProRoot MTA to improve handling characteristics and decrease setting time. J Endod 2007;33:1231-1234.

10 Atmeh AR, Chong EZ, Richard G, et al: Dentin-cement interfacial interaction: calcium silicates and polyalkenoates. J Dent Res 2012; 91:454-459.

11 Gancedo-Caravia L, Garcia-Barbero E: Influence of humidity and setting time on the push-out strength of mineral trioxide aggregate obturations. J Endod 2006;32:894-896.

12 Wang Z, Ma J, Shen Y, et al: Acidic pH weakens the microhardness and microstructure of three tricalcium silicate materials. Int Endod J 2015;48:323-332.

13 Nekoofar MH, Aseeley Z, Dummer PM: The effect of various mixing techniques on the surface microhardness of mineral trioxide aggregate. Int Endod J 2010;43:312-320.

14 Hong ST, Bae KS, Baek SH, et al: Effects of root canal irrigants on the push-out strength and hydration behavior of accelerated mineral trioxide aggregate in its early setting phase. J Endod 2010;36:1995-1999.
15 Akcay H, Arslan H, Akcay M, Mese M, Sahin $\mathrm{NN}$ : Evaluation of the bond strength of rootend placed mineral trioxide aggregate and Biodentine in the absence/presence of blood contamination. Eur J Dent 2016;10:370-375.

16 Guneser MB, Akbulut MB, Eldeniz AU: Effect of various endodontic irrigants on the pushout bond strength of Biodentine and conventional root perforation repair materials. J Endod 2013;39:380-384.

17 Camilleri J, Sorrentino F, Damidot D: Characterization of un-hydrated and hydrated BioAggregate $^{\mathrm{TM}}$ and MTA Angelus ${ }^{\mathrm{TM}}$. Clin Oral Investig 2015;19:689-698.

18 Oh S, Perinpanayagam H, Lee Y, et al: Effect of acidic solutions on the microhardness of dentin and set OrthoMTA and their cytotoxicity on murine macrophage. Restor Dent Endod 2016;41:12-21.

19 Alsubait SA, Hashem Q, AlHargan N, et al: Comparative evaluation of push-out bond strength of ProRoot MTA, Bioaggregate and Biodentine. J Contemp Dent Pract 2014;15: 336-340.

20 Dawood AE, Manton DJ, et al: Push-out bond strength of CPP-ACP-modified calcium silicate-based cements. Dent Mater J 2015;34: 490-494.

21 Han L, Kodama S, Okiji T: Evaluation of calcium-releasing and apatite-forming abilities of fast-setting calcium silicate-based endodontic materials. Int Endod J 2015;48:124-130. 
22 Camilleri J, Sorrentino F, Damidot D: Investigation of the hydration and bioactivity of radiopacified tricalcium silicate cement, Biodentine and MTA Angelus. Dent Mater 2013; 29:580-593.

23 Han L, Okiji T: Bioactivity evaluation of three calcium silicate-based endodontic materials. Int Endod J 2013;46:808-814.

24 Grech L, Mallia B, Camilleri J: Investigation of the physical properties of tricalcium silicate cement-based root-end filling materials. Dent Mater 2013;29:e20-e28.
25 Pane ES, Palamara JE, Messer HH: Critical evaluation of the push-out test for root canal filling materials. J Endod 2013;39:669-673.

26 Shokouhinejad N, Nekoofar MH, Iravani A, et al: Effect of acidic environment on the push-out bond strength of mineral trioxide aggregate. J Endod 2010;36:871-874.

27 Rached-Junior FJ, Souza-Gabriel AE, Alfredo E, Miranda CE, Silva-Sousa YT, Sousa-Neto MD: Bond strength of Epiphany sealer prepared with resinous solvent. J Endod 2009;35: 251-255.
28 Leloup G, D'Hoore W, Bouter D, et al: Metaanalytical review of factors involved in dentin adherence. J Dent Res 2001;80:1605-1614.

29 Bolhari B, Nekoofar MH, Sharifian M, et al: Acid and microhardness of mineral trioxide aggregate and mineral trioxide aggregate-like materials. J Endod 2014;40:432-435.

30 Caronna V, Himel V, Yu Q, et al: Comparison of the surface hardness among 3 materials used in an experimental apexification model under moist and dry environments. J Endod 2014;40:986-989. 